\title{
Cholesterol Efflux and Reverse Cholesterol Transport
}

\author{
Elda Favari, Angelika Chroni, Uwe J.F. Tietge, Ilaria Zanotti, \\ Joan Carles Escolà-Gil, and Franco Bernini
}

\section{Contents}

1 Cholesterol Efflux as the First Step of Reverse Cholesterol Transport (RCT) . ........ 183

$1.1 \quad$ ABCA1-Mediated Lipid Efflux to Lipid-Poor apoA-I ...................... 184

1.2 Cholesterol Efflux to Lipidated HDL ................................. 185

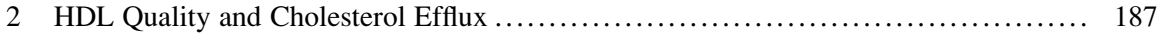

3 RCT in Animal Models ................................................... 190

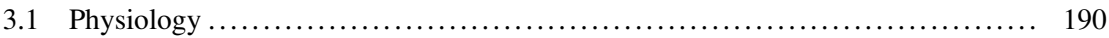

3.1.1 Methodological Approaches to Quantify RCT In Vivo ................ 191

3.1.2 Factors Impacting In Vivo RCT ............................... 192

3.2 Pharmacology ...................................................... 196

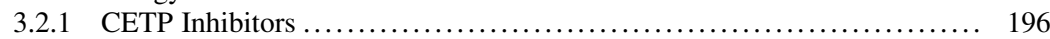

3.2.2 Nuclear Receptor Activation ................................. 197

3.2.3 Cholesterol Absorption Inhibitors ............................... 197

3.2.4 Augmenting or Mimicking apoA-I ........................... 197

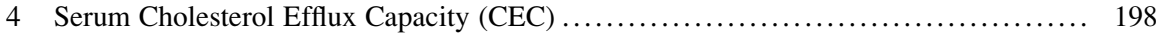

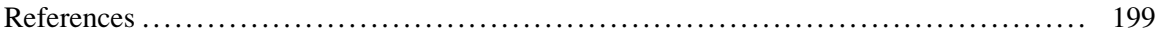

E. Favari • I. Zanotti • F. Bernini $(\bowtie)$

Department of Pharmacy, University of Parma, Parco Area delle Scienze 27/A, 43124 Parma, Italy e-mail: fbernini@unipr.it

A. Chroni

Institute of Biosciences and Applications, National Center for Scientific Research "Demokritos", Agia Paraskevi, Athens, Greece

U.J.F. Tietge

Department of Pediatrics, University of Groningen, University Medical Center Groningen,

Groningen, The Netherlands

J.C. Escolà-Gil

IIB Sant Pau-CIBER de Diabetes y Enfermedades Metabolicas Asociadas, Barcelona, Spain

(C) The Author(s) 2015

A. von Eckardstein, D. Kardassis (eds.), High Density Lipoproteins, Handbook of

Experimental Pharmacology 224, DOI 10.1007/978-3-319-09665-0_4 


\title{
Abstract
}

Both alterations of lipid/lipoprotein metabolism and inflammatory events contribute to the formation of the atherosclerotic plaque, characterized by the accumulation of abnormal amounts of cholesterol and macrophages in the artery wall. Reverse cholesterol transport (RCT) may counteract the pathogenic events leading to the formation and development of atheroma, by promoting the highdensity lipoprotein (HDL)-mediated removal of cholesterol from the artery wall. Recent in vivo studies established the inverse relationship between RCT efficiency and atherosclerotic cardiovascular diseases (CVD), thus suggesting that the promotion of this process may represent a novel strategy to reduce atherosclerotic plaque burden and subsequent cardiovascular events. HDL plays a primary role in all stages of RCT: (1) cholesterol efflux, where these lipoproteins remove excess cholesterol from cells; (2) lipoprotein remodeling, where HDL undergo structural modifications with possible impact on their function; and (3) hepatic lipid uptake, where HDL releases cholesterol to the liver, for the final excretion into bile and feces. Although the inverse association between HDL plasma levels and CVD risk has been postulated for years, recently this concept has been challenged by studies reporting that HDL antiatherogenic functions may be independent of their plasma levels. Therefore, assessment of HDL function, evaluated as the capacity to promote cell cholesterol efflux may offer a better prediction of CVD than HDL levels alone. Consistent with this idea, it has been recently demonstrated that the evaluation of serum cholesterol efflux capacity (CEC) is a predictor of atherosclerosis extent in humans.

\section{Keywords}

$\mathrm{HDL} \cdot \mathrm{RCT} \bullet$ Cholesterol efflux $\bullet \mathrm{CEC}$

\author{
Abbreviations \\ 15-LO-1 15-Lipoxygenase-1 \\ ABCA1 ATP-binding cassette transporter A1 \\ ABCG1 ATP-binding cassette transporter G1 \\ apoB Apolipoprotein B \\ apoE Apolipoprotein E \\ CEC Cholesterol efflux capacity \\ CETP Cholesteryl ester transfer protein \\ CVD Cardiovascular diseases \\ EL Endothelial lipase \\ HDL High-density lipoproteins \\ LCAT Lecithin-cholesterol acyltransferase
}




$\begin{array}{ll}\text { LDL } & \text { Low-density lipoproteins } \\ \text { LXR } & \text { Liver X receptor } \\ \text { LXR/RXR } & \text { Liver X receptor/retinoid X receptor } \\ \text { MPO } & \text { Myeloperoxidase } \\ \text { NPC1L1 } & \text { Niemann-Pick type C like } 1 \\ \text { PLA } 2 & \text { Phospholipase } A_{2} \\ \text { PLTP } & \text { Phospholipid transfer protein } \\ \text { PPAR } & \text { Peroxisome proliferator-activated receptor } \\ \text { RCT } & \text { Reverse cholesterol transport } \\ \text { SR-BI } & \text { Scavenger receptor class B type I }\end{array}$

\section{Cholesterol Efflux as the First Step of Reverse Cholesterol Transport (RCT)}

Lipid accumulation within macrophages, leading to the formation of foam cells, represents a main feature of atherogenesis (Wynn et al. 2013). Among the strategies by which cells may counteract this process, the release of excess cholesterol to extracellular lipid acceptors has been matter of several studies in the recent years. Although cholesterol efflux from macrophages has a minor contribution to the whole body cholesterol transported by plasma high-density lipoproteins (HDL), it is the most important for the antiatherosclerotic extent. For this reason, the macrophage RCT concept has been proposed, and it was recently identified as a novel therapeutic target (Rosenson et al. 2012).

Both active and passive mechanisms are responsible for cholesterol efflux, and several components, such as cell cholesterol status, lipid transporter activity, and the nature of extracellular acceptors, have been shown to impact its efficiency (Zanotti et al. 2012).

The efflux process involves cholesterol localized on the plasma membrane that in turn may derive from intracellular sites, such as the late endosomal/lysosomal compartment and from the Golgi apparatus. Cholesterol localized in the endoplasmic reticulum originates from endogenous synthesis and can be delivered to intracellular organelles mainly through a nonvesicular pathway involving protein carriers. Cholesterol in endosomes and lysosomes derives from lipoprotein uptake and undergoes hydrolyzation by acid hydrolase (Maxfield and Tabas 2005). This lipoprotein-derived cholesterol is then rapidly released into the cytoplasm and delivered throughout the cells by Niemann-Pick type $\mathrm{C} 1$ and 2 . These proteins, expressed on the membrane of late endosomes and in lysosomes, respectively, facilitate lipid mobilization from these compartments to the plasma membranes via the trans-Golgi network (Boadu and Francis 2006). In physiological conditions cholesterol content in intracellular locations is low, because most of it (about $80 \%$ ) is transferred to the plasma membrane, where it establishes a dynamic equilibrium with endoplasmic reticulum and the Golgi system pools (Huang et al. 2003). The 
plasma membrane bilayer contains distinct lipid environments in a steady state equilibrium. Lipid rafts are characterized by a tightly packed, liquid-ordered state, where cholesterol associates with sphingolipids and caveolin, playing a crucial role in cell signaling (Gargalovic and Dory 2003). Several studies carried out in macrophages, demonstrated that the major mechanisms of cholesterol efflux are raft-independent (Gargalovic and Dory 2003). The non-raft membrane microdomains serve as the principal source of cholesterol available for interaction with extracellular acceptors and subsequent efflux via ATP-binding cassette transporter A1 (ABCA1) that is localized in these portions of the membrane (Landry et al. 2006; Vaughan and Oram 2005). Interestingly, RCT can be triggered also from extracellular matrix-associated cholesterol microdomains, whose formation is mediated by ATP-binding cassette transporter G1 (ABCG1) (Freeman et al. 2013).

Mechanisms accounting for cholesterol efflux include passive diffusion processes as well as active pathways mediated by ABCA1, ABCG1, and scavenger receptor class B type I (SR-BI). Aqueous diffusion mainly involves free cholesterol in the plasma membrane and HDL as lipid acceptors. The nature of this process is a matter of debate: whereas this mechanism appears to be a relevant contributor of lipid removal in foam cell macrophages (Adorni et al. 2007), the involvement of a still unknown transporter is not completely ruled out.

\subsection{ABCA1-Mediated Lipid Efflux to Lipid-Poor apoA-I}

ABCA1 is a 2,261-amino acid integral membrane protein, member of the large superfamily of ABC transporters that use ATP as an energy source to transport lipids across membranes. $\mathrm{ABC}$ transporters are characterized by the presence of nucleotide-binding domains containing two conserved peptide motifs known as Walker A and Walker B, a unique amino acid signature between the two Walker motifs, which defines the family. ABC transporters are integrated into the membrane by domains containing six transmembrane helices. The minimum requirement for an active $\mathrm{ABC}$ transporter is two nucleotide-binding and two 6-helix transmembrane domains (Oram and Lawn 2001). At the cellular level ABCA1 is localized both on the plasma membrane and intracellular compartments, the Golgi complex, and the late endosome/lysosomes, cycling between these loci and promoting a flow of intracellular cholesterol from the late endosomes/lysosomes, through the trans-Golgi complex, to the plasma membrane (Boadu and Francis 2006). This movement produces an ABCA1-dependent depletion of intracellular pools of cholesterol that affects both the "regulatory" pool of cholesterol and the LDL-derived cholesterol. These activities result in the modulation of a number of cellular events including: endogenous cholesterol synthesis, LDL receptor expression, cholesteryl ester turnover, and nascent HDL formation (Boadu and Francis 2006). The last process is driven by ABCA1-mediated removal of lipids from the cell membrane to extracellular acceptors represented by lipid-free or lipid-poor apolipoproteins. ABCA1 expression on the plasma membrane leads to the generation of non-raft microdomains and enlargement of cholesterol and phospholipid 
domains in the outer leaflet, thus facilitating the interaction with apoA-I and subsequent cholesterol efflux. It is worth noting that these pools of cholesterol created by ABCA1 are specific substrates for ABCA1-mediated lipid release because they selectively interact with lipid-free or lipid-poor apolipoproteins (Landry et al. 2006). ABCA1 exerts its function through a floppase activity, which drives cholesterol and phospholipids from the inner leaflet of the membrane to cell surface domains and eventually back to intracellular compartments. In addition, ABCA1 promotes vesicular trafficking of cholesterol, phospholipids and ABCA1 itself between the plasma membrane and intracellular compartments (Landry et al. 2006). Cholesterol efflux via ABCA1 is related to the protein level, in turn regulated by transcriptional or posttranslational mechanisms. Abca1 gene expression is primarily induced by the stimulation of liver $\mathrm{X}$ receptor/retinoid $\mathrm{X}$ receptor (LXR/RXR) axis, stimulated by cholesterol accumulation in the cells (Larrede et al. 2009). Recently, the role of microRNAs in the inhibitory control of Abcal expression has emerged (Sun et al. 2012; Rayner et al. 2010; Ramirez et al. 2011). The posttranscriptional regulation includes mechanisms that involve (1) the stabilization of ABCA1 protein by apoA-I and (2) the acceleration of its turnover by calpain-mediated proteolysis or polyunsaturated fatty acids (Oram and Vaughan 2006).

\subsection{Cholesterol Efflux to Lipidated HDL}

ABCA1-mediated generation of nascent HDL particles may in turn promote cholesterol efflux via ABCG1 and SR-BI. The former is a half-size ABC protein, where the nucleotide-binding domain at the N-terminus is followed by six transmembrane-spanning domains. Interestingly, various transcripts of ABCG1 have been detected in different cells, possibly arising from alternative splicing events (Schmitz et al. 2001). Early studies reported many similarities between ABCG1 and ABCA1, including the cellular localization, the translocation from intracellular compartments to the plasma membrane, the floppase activity, and the expression promoted by cholesterol enrichment via LXR. A main feature of ABCG1-mediated efflux is the specific interaction with HDL and low-density lipoproteins (LDL) thus accounting for the elimination of cholesterol and toxic oxysterols from the macrophages (Vaughan and Oram 2005). It is important to note that under physiologic conditions $\mathrm{ABCA} 1$ and $\mathrm{ABCG} 1$ can act in a sequential fashion, with ABCA1 generating, which then promote lipid release via ABCG1 (Gelissen et al. 2006). However, recent data challenge these concepts, suggesting that, unlike ABCA1, ABCG1 effluxes cellular cholesterol by a process that is not dependent upon interaction with an extracellular protein (Tarling and Edwards 2012).

Although several cholesterol-responsive ABC transporters, other than ABCA1 and $A B C G 1$, have been described in the macrophages, their potential relevance for the process of foam cell formation and RCT needs further investigations (Fu et al. 2013). 
SR-BI is an $82-\mathrm{kDa}$ integral membrane protein, belonging to the CD36 family, whose physiological role is related to the selective uptake of HDL cholesteryl ester, the process by which the core cholesteryl ester is taken into the cell without the endocytic uptake and degradation of the whole HDL. Since this pathway is the major route for the delivery of HDL cholesteryl ester to the liver, the role of SR-BI in determining the plasma levels of HDL is of major importance. Importantly, SR-BI has shown to stimulate free cholesterol efflux facilitating the aqueous diffusion pathway to phospholipid-enriched acceptors. Thus, the expression of this receptor establishes a bidirectional flux between cells and HDL, whose net effect will be related to cell cholesterol status, as well as the composition and concentration of the acceptor in the extracellular environment. Differently from the $\mathrm{ABC}$ transporters, SR-BI is localized in caveolae, a subset of lipid rafts, cell surface invaginations enriched in free cholesterol (Rosenson et al. 2012). Despite the significant role of SR-BI in cell cholesterol metabolism, its role in cholesterol efflux from macrophages is still unclear. It is important to note that the relative contribution of a single pathway to cholesterol export is species specific: whereas ABCA1-mediated efflux is the predominant mechanism both in human and murine cultured foam cells, the role of ABCG1 is elusive in the former, but not in the latter. Conversely, SR-BI (Cla-1 in humans) plays a pivotal role in human, but not murine cells (Adorni et al. 2007; Sun et al. 2012). Moreover, the antiatherosclerotic properties of $\mathrm{ABCA} 1$ and $\mathrm{ABCG} 1$ have recently been challenged by the demonstration that some efflux-independent activities could be related to deleterious effects on macrophage function (Adorni et al. 2011; Olivier et al. 2012).

A relevant role in cholesterol efflux from macrophages and RCT has been also attributed to apolipoprotein E (apoE) (Zanotti et al. 2011a, b). This 34-kDa protein is synthesized by many cell types, including macrophages, upon different stimuli, such as differentiation, cytokines, and lipid enrichment. The last process activates the LXR pathway, as described for ABCA1 and ABCG1. ApoE synthesis in the endoplasmic reticulum is followed by its movement to the Golgi and trans-Golgi network and incorporation into vesicular structures, before being transported to the plasma membrane for the final secretion. Secreted apoE can be released into the extracellular medium or alternatively can bind to the cell surface, particularly in association with heparan sulfate proteoglycans. Cell surface pools may be reinternalized and subsequently degraded, transported to the Golgi network for posttranslational modifications, or released into the extracellular medium (Kockx et al. 2008). When ApoE is secreted from cholesterol-enriched macrophages, this process can promote cholesterol efflux in the absence of added cholesterol acceptors or in presence of exogenous HDL, causing the generation of nascent HDL particles. There is evidence that apoE can promote cholesterol release by both ABCA1-dependent and independent mechanisms, whereas ABCG1 may contribute by driving cholesterol efflux to apoE-enriched particles (Huang et al. 2001). 


\section{$2 \quad$ HDL Quality and Cholesterol Efflux}

HDL comprises several subclasses that differ in composition and size and exhibit a series of atheroprotective and other properties, including the ability to efflux cholesterol from various cell types, as well as antioxidative, anti-inflammatory, anticoagulatory, and anti-aggregatory properties (Annema and von Eckardstein 2013; Vickers and Remaley 2014). These properties are exerted by the various protein and lipid components of HDL (Annema and von Eckardstein 2013; Vickers and Remaley 2014). It is increasingly accepted that the quality rather than quantity of HDL is more relevant for its atheroprotective activity (see chapter "Dysfunc tional HDL: From Structure-Function-Relationships to Biomarkers" for more details).

As anticipated, the ability of HDL to promote cholesterol efflux from lipid-laden macrophages is thought to be important for the atheroprotective function of HDL. The capacity of HDL to promote cholesterol efflux from macrophages was shown to have a strong inverse association with both carotid intima-media thickness and the likelihood of angiographic coronary artery disease, independently of the HDL-C level (Khera et al. 2011). As mentioned before, cellular cholesterol efflux is mediated by a number of pathways, with the various HDL subpopulations to display a varied capacity to promote cholesterol efflux via each of these pathways (Annema and von Eckardstein 2013; Vickers and Remaley 2014). Therefore, the efficiency of serum from an individual to accept cellular cholesterol can be affected by the distribution and composition of HDL particles acting as cholesterol acceptors. Indeed, it has been shown that apolipoprotein B (apoB)-depleted sera from subjects with similar HDL-C or apoA-I can have higher total macrophage efflux capacity due to significantly higher ABCA1-mediated efflux, and this efflux is significantly correlated with the levels of pre $\beta 1-H D L$ (de la Llera-Moya et al. 2010; Calabresi et al. 2009). Small discoidal pre $\beta 1-H D L$ particles are also efficient acceptors of cell cholesterol via ABCG1 (Favari et al. 2009). In another study, apoB-depleted sera from patients treated with the cholesteryl ester transfer protein (CETP) inhibitor anacetrapib were shown to have enhanced ability to promote ABCG1-mediated cholesterol efflux from macrophages, which was associated with increased lecithincholesterol acyltransferase (LCAT) and apoE mass in HDL (Yvan-Charvet et al. 2010).

The various components of HDL particles can undergo structural or chemical modifications during atherogenesis or other pathologic processes, having as a result adverse effects on HDL functionality, including the cholesterol efflux capacity. In vitro and in vivo studies have shown that enzymatic oxidation, lipolysis, and proteolysis can modify HDL and affect the HDL capacity to promote cellular cholesterol efflux. HDL isolated from humans with established CVD was found to contain higher levels of protein-bound 3-chlorotyrosine and protein-bound 3-nitrotyrosine compared to HDL from controls (Bergt et al. 2004; Pennathur et al. 2004; Zheng et al. 2004). In addition, the levels of both 3-chlorotyrosine and 3-nitrotyrosine were higher in HDL isolated from atherosclerotic lesions compared with plasma HDL. One pathway that generates such species involves 
myeloperoxidase (MPO), a major constituent of artery wall macrophages (Bergt et al. 2004; Pennathur et al. 2004; Zheng et al. 2004). It was proposed that apoA-I is a selective target for MPO-catalyzed nitration and chlorination in vivo and that MPO-catalyzed oxidation of HDL and apoA-I results in selective inhibition of ABCA1-dependent cholesterol efflux from macrophages (Zheng et al. 2004). More specifically, it was shown that chlorination of apoA-I impaired the ability of the protein to promote cholesterol efflux by MPO, while nitration had a much lesser effect (Bergt et al. 2004; Zheng et al. 2005; Shao et al. 2005) and that a combination of Tyr-192 chlorination and methionine oxidation is necessary for depriving apoA-I of its ABCA1-dependent cholesterol transport activity (Shao et al. 2006). More recently it was suggested that oxidative damage to apoA-I by MPO limits the ability of apoA-I to be liberated in a lipid-free form from HDL and that this impairment of apoA-I exchange reaction may contribute to reduced ABCA1-mediated cholesterol efflux (Cavigiolio et al. 2010).

Treatment of $\mathrm{HDL}_{3}$ with the reticulocyte-type 15-lipoxygenase-1 (15-LO-1), which has been suggested to play a pathophysiological role in atherosclerosis, induced HDL apolipoprotein cross-linking and reduced cholesterol efflux from lipid-laden J774 cells (Pirillo et al. 2006). A reduced binding of 15-LO-modified $\mathrm{HDL}_{3}$ to SR-BI explained, in part, the observed reduction of cholesterol efflux. In addition, the ABCA1-mediated cholesterol efflux was also reduced, as a consequence of loss of pre $\beta$-particles after $\mathrm{HDL}_{3}$ modification (Pirillo et al. 2006).

The capacity of HDL to promote cellular cholesterol efflux from lipid-loaded mouse peritoneal macrophages was significantly decreased after treatment of HDL with secretory phospholipase $\mathrm{A}_{2}$ ( $\mathrm{PLA}_{2}$ ) group X or V (Zanotti et al. 2011a, b). $\mathrm{SPLA}_{2}-\mathrm{X}$ and $\mathrm{sPLA}_{2}-\mathrm{V}$ that have been associated with the pathogenesis of atherosclerosis affect the capacity of HDL to promote cholesterol efflux by catalyzing the hydrolysis of phosphatidylcholine in HDL without any modification of apoA-I (Ishimoto et al. 2003).

Several proteases, including metalloproteinases, cathepsins, chymase, tryptase, kallikrein, neutrophil elastase, and plasmin, that are secreted from various types of cells of human atherosclerotic arterial intima can proteolytically modify HDL in vitro (Lee-Rueckert et al. 2011). It has been shown that the various proteases proteolyze apoA-I that is present in pre $\beta$-HDL and therefore reduce cholesterol efflux from macrophage foam cells (Favari et al. 2004). A series of studies aiming to elucidate the effect of proteolytic modification of HDL on cholesterol efflux and RCT used mast cells and the neutral serine proteases chymase and tryptase, which are secreted from mast cells. Treatment of $\mathrm{HDL}_{3}$ with human chymase resulted in rapid depletion of pre $\beta$-HDL and a concomitant decrease in the efflux of cholesterol and phospholipids by an ABCA1-dependent pathway, while aqueous or SR-BIfacilitated diffusion of cholesterol was not affected (Favari et al. 2004). Furthermore, local activation of mast cells by the specific mast cell degranulating compound 48/80 in the mouse peritoneal cavity, which causes acute release of active chymase, resulted in a $90 \%$ reduction of human apoA-I injected into the peritoneal cavity. This reduction reflected the reduction in pre $\beta$-HDL particles and resulted in attenuation of cholesterol efflux from intraperitoneally co-injected J774 
macrophages and a reduced rate of macrophage RCT. In addition, pretreatment of apoA-I with chymase also fully abolished the stimulatory effect of untreated apoA-I to promote the transfer of macrophage-derived cholesterol to the intestine (Lee-Rueckert, Silvennoinen et al. 2011).

Proteolysis of lipid-free apoA-I or pre $\beta$-HDL- or $\mathrm{HDL}_{3}$-associated apoA-I by intima proteases may result in the formation of carboxy-terminal truncated apoA-I fragments. Specifically, it has been shown recently that lipid-free apoA-I is preferentially digested by chymase at the $\mathrm{C}$-terminus rather than the $\mathrm{N}$-terminus and that the $\mathrm{Phe}_{229}$ and $\mathrm{Tyr}_{192}$ residues are the main cleavage sites, while the $\mathrm{Phe}_{225}$ residue is a minor cleavage site (Usami et al. 2013). C-terminally truncated apoA-I was detected in normal human serum using a specific monoclonal antibody (16-4mAb) recognizing C-terminally truncated apoA-I that has been cleaved after $\mathrm{Phe}_{225}$ by chymase (Usami et al. 2011). In addition, it has been shown that proteolysis of apoA-I in pre $\beta$-HDL by plasmin generated apoA-I fragments lacking the C-terminal region (Kunitake et al. 1990). Such C-terminal truncation of apoA-I can affect its capacity to promote ABCA1-mediated cholesterol efflux and the biogenesis of HDL. Previous studies showed that when C-terminal segments that contain residues 220-231 are deleted, the apoA-I cannot associate with ABCA1 and has a diminished capacity to promote ABCA1-mediated phospholipid cholesterol efflux (Favari et al. 2002; Chroni et al. 2003, 2004). In another study, the treatment of lipid-free human apoA-I by chymase-containing lysate derived from mouse peritoneal mast cells or recombinant human chymase generated apoA-I fragments, lacking the C-terminal site (Usami et al. 2013), which had diminished capacity to promote cholesterol efflux from mouse peritoneal macrophage foam cells (Lee-Rueckert, Silvennoinen et al. 2011). Furthermore, adenovirus-mediated gene transfer in apoA-I-deficient mice showed that the apoA-I mutants that lack C-terminal residues 220-231 fail to form spherical $\alpha H D L$ in vivo (Chroni et al. 2003, 2007).

Other proteolysis studies showed that apoA-I in $\mathrm{HDL}_{3}$ by various recombinant metalloproteinases in vitro or by metalloproteinases secreted from macrophages generated apoA-I fragments also lacking the carboxyl-terminal region (cleavage after residues 213, 202, 215, 225,199, 191, or 188) (Lindstedt et al. 1999; Eberini et al. 2002). In addition, chymase, in vitro, cleaves apoA-I in reconstituted HDL at the C-terminus (after $\mathrm{Phe}_{225}$ ) (Lee et al. 2003). These C-terminal truncated lipoprotein-associated apoA-I displayed diminished capacity to promote cholesterol efflux from mouse macrophage foam cells (Lindstedt et al. 1999; Lee et al. 2003). A recent study showed that reconstituted HDL containing the C-terminal deletion mutants apoA-I $(\Delta(185-243))$ or apoA-I $(\Delta(220-243))$ had strongly impaired capacity to promote ABCG1-mediated cholesterol efflux (Daniil et al. 2013). In addition, limited proteolysis of rHDL containing wild-type apoA-I by plasmin resulted in $66 \%$ decrease of ABCG1-dependent cholesterol efflux (Daniil et al. 2013). It is therefore possible that proteolysis of HDL-associated apoA-I in vivo by proteases, which are present in the human arterial intima, could yield apoA-I fragments similar to the apoA-I $(\Delta(185-243))$ or apoA-I $(\Delta(220-243))$ mutants and thus may impair their capacity to promote ABCG1-mediated 
cholesterol efflux from macrophages. The structure-function relationship seen in this study between rHDL-associated apoA-I mutants and ABCG1-mediated cholesterol efflux closely resembles that seen before in lipid-free apoA-I mutants and ABCA1-dependent cholesterol efflux, suggesting that both processes depend on the same structural determinants of apoA-I.

The levels, composition, and the antiatherogenic properties, including the cholesterol efflux capacity, of HDL has been shown to be affected in patients suffering from chronic inflammatory rheumatic diseases (Onat and Direskeneli 2012; Watanabe et al. 2012). Specifically, cholesterol efflux capacity of HDL was impaired in rheumatoid arthritis and systemic lupus erythematosus patients with high disease activity and was correlated with systemic inflammation, higher plasma MPO activity, and HDL's antioxidant capacity (Ronda et al. 2013; CharlesSchoeman et al. 2012). In addition, HDL cholesterol efflux capacity was lower in patients with psoriasis (Watanabe et al. 2012; Holzer et al. 2013), while antipsoriatic therapy significantly improved the HDL cholesterol efflux capacity, along with improved serum LCAT activity and without any effect on serum HDL cholesterol levels (Holzer et al. 2013).

Overall, modifications of HDL particles have been demonstrated to affect the atheroprotective properties of HDL, including its capacity to promote cellular cholesterol efflux. Elucidation of the conditions and processes that affect the compositional, structural, and functional intactness of HDL may become an important tool for the assessment of cardiovascular risk and may provide us with novel therapeutic approaches. In addition, monitoring of the capacity of HDL to promote cholesterol efflux could be useful to evaluate novel therapeutic approaches for the reduction of cardiovascular risk, as it will be discussed later.

\section{$3 \quad$ RCT in Animal Models}

\subsection{Physiology}

The term RCT summarizes the transport of cholesterol from macrophage foam cells within atherosclerotic lesions through the aqueous compartment of the blood for final excretion into the feces, which could either occur directly as cholesterol or after metabolic conversion into bile acids (Annema and Tietge 2012). As detailed above, cholesterol efflux from lipid-laden macrophages is mediated by the transporters ABCA1, ABCG1, and SR-BI with HDL as acceptor, and this process is facilitated by apoE. Within the blood HDL can be remodeled in several ways. LCAT esterifies free cholesterol in the particles resulting in larger HDL and increased plasma HDL-C levels (Annema and Tietge 2012). CETP transfers cholesterol out of HDL toward apoB-containing lipoproteins in exchange for triglycerides, which are then rapidly hydrolyzed by hepatic lipase (HL) (Annema and Tietge 2012). Thereby CETP and also HL, either alone or in combination, lower plasma HDL-C levels. Other HDL remodeling proteins that increase the catabolic rate of HDL and lower circulating HDL-C are the phospholipid transfer 
protein (PLTP) (Annema and Tietge 2012) and the phospholipases endothelial lipase (EL) (Annema and Tietge 2011) and group IIA secretory phospholipase $\mathrm{A}_{2}$

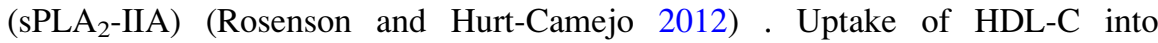
hepatocytes can be accomplished in two distinct ways, via selective uptake mediated by SR-BI (Annema and Tietge 2012) or via holoparticle uptake in a process that has not been fully elucidated yet (Vantourout et al. 2010). However, it has been shown that via ectopic localization of the mitochondrial F1-beta-ATPase on the cell membrane, ADP is generated which then stimulates via the ADP receptor P2Y13 holoparticle uptake of HDL by still elusive receptor mechanisms; thereby, HDL holoparticle uptake is fully dependent on the expression of the ADP receptor P2Y13 (Vantourout et al. 2010). In hepatocytes, cholesterol is either secreted directly into the bile, which is mediated in terms of mass mainly by ABCG5/G8 and to a lesser extent by SR-BI, or following conversion into bile acids, for which $\mathrm{ABCB} 11$ is the critical transporter into bile (Dikkers and Tietge 2010). Cholesterol can be reabsorbed in the intestine by Niemann-Pick type $C$ like 1 (NPC1L1) but also (re)secreted by the ABCG5/G8 heterodimer, whereby a low activity of NPC1L1 would increase and a low activity of ABCG5/G8 would decrease cholesterol excretion into the feces (Annema and Tietge 2012). Bile acids can be reabsorbed in the terminal ileum by ASBT (Dawson 2011). In addition to its role in sterol absorption, the intestine has also been indicated to mediate direct secretion of cholesterol, a process termed transintestinal cholesterol excretion [TICE, current knowledge summarized in Tietge and Groen (2013)]. All different steps discussed in this paragraph have the potential to impact on and modulate RCT.

\subsubsection{Methodological Approaches to Quantify RCT In Vivo}

The key methodological problem for quantifying RCT specifically from macrophages, which is most relevant for atherosclerotic disease, is that the macrophage cholesterol pool is rather small. Initially employed techniques such as mass determinations of centripetal cholesterol fluxes to the liver or isotope dilution methods were therefore not able to accurately allow a conclusion of specific cholesterol fluxes from macrophages (Annema and Tietge 2012). Recently, a now widely used and accepted technique had been developed that overcomes such methodological drawbacks (Zhang et al. 2003). Thereby, macrophages, either primary or cell lines, are loaded with radiolabeled cholesterol and then injected intraperitoneally into recipient animals. After injection, the appearance of the tracer is determined in plasma at different time points and, most importantly, in the feces that are collected continuously. The time course of such experiments is usually 24-48 $\mathrm{h}$; in the feces distinguishing between labels in neutral sterols and bile acids is in our view preferable.

Although this method allows tracing of cholesterol from macrophages to feces, there are certain limitations that need to be taken into account when interpreting results from such studies. One is that in the assay a potential influx of cholesterol from the plasma compartment into the macrophage is not taken into account. As first steps to overcome this, two approaches were reported, in which the application procedure of the labeled macrophages is modified in a way that allows reisolation of 
the cells to measure label as well as mass cholesterol content. One of these approaches makes use of Matrigel plugs that are implanted subcutaneously (Malik and Smith 2009), while the other another, entrapment of the macrophages in semipermeable holofibers (Weibel et al. 2011). Another limitation of in vivo RCT assays is that all approaches place the labeled macrophages outside the vascular compartment, which supposedly is a good surrogate but might also not accurately reflect the situation in an atherosclerotic lesion in terms of oxygen tension, $\mathrm{pH}$, or accessibility by the HDL particles. In the following, results obtained from the above-described method will be summarized. It is relevant to point out that although initial steps toward a macrophage RCT assay in humans have been reported at conferences (Dunbar et al. 2013), current knowledge on the regulation of in vivo RCT is based on studies in animal models that differ in several aspects of their lipoprotein metabolism.

\subsubsection{Factors Impacting In Vivo RCT}

Looking at the level of the macrophage, available studies consistently indicate that expression of ABCA1 and ABCG1 is associated with increased RCT (Wang et al. 2007a, b; Out et al. 2008). However, SR-BI deficiency in macrophages does not impact RCT (Wang et al. 2007b; Zhao et al. 2011). On the other hand, macrophage apoE was shown to stimulate RCT (Zanotti et al. 2011a, b).

Regarding plasma proteins that have a role in HDL metabolism, for LCAT surprisingly no consistent effects on RCT were observed using several different models and overexpression as well as knockout strategies resulting in the conclusion that LCAT only minimally contributes to RCT although it has a major role in determining plasma HDL-C levels (Tanigawa et al. 2009). In the case of the lipid transfer proteins, PLTP overexpression resulted in increased RCT (Samyn et al. 2009), while for CETP (over)expressing models, either an increase (Tchoua et al. 2008; Tanigawa et al. 2007), dependent on functional expression of the LDL receptor (Tanigawa et al. 2007), or no effect (Rotllan et al. 2008) was observed. With respect to (phospho)lipases, SPLA $_{2}$-IIA had no impact on RCT (Annema et al. 2010). In case of HL and EL, conflicting data have been reported. While one group found no effect of knocking out either lipase separate or both in combination (Brown et al. 2010), others reported increased RCT for EL as well as HL (Escola-Gil et al. 2013). In contrast to apoE expression in macrophages, systemic overexpression of apoE had no effect on RCT either in wild-type or CETP transgenic mice (Annema et al. 2012).

In the case of the hepatic component of RCT, SR-BI expression in hepatocytes has a clear increasing effect on RCT as indicated in studies using both knockout and overexpression (Zhao et al. 2011; El Bouhassani et al. 2011; Zhang et al. 2005). Interestingly, it has been shown that in the liver the impact of SR-BI is independent of ABCG5/G8 expression, since double-knockout mice for both transporters had a significant decrease in RCT compared with ABCG5 knockout mice alone (Dikkers et al. 2013). ABCG5/G8 knockouts on the other hand exhibited no change in RCT (Calpe-Berdiel et al. 2008). In addition, also the consequences of abolishing functional HDL holoparticle uptake into hepatocytes on RCT were assessed. In 
$\mathrm{P}_{2} \mathrm{Y}_{13}$ knockout mice, this pathway is completely absent translating into a significant reduction in macrophage-to-feces RCT (Fabre et al. 2010; Lichtenstein et al. 2013). These combined studies indicate that both selective uptake and holoparticle uptake of HDL are critical for RCT. Regarding expression of ABCA1 in hepatocytes, liver supposedly contributes around $70 \%$ to total HDL formation (Timmins et al. 2005); the picture is not so clear. While in wild-type and SR-BI knockout mice blocking hepatic ABCA1 with probucol enhanced RCT (Annema et al. 2012; Yamamoto et al. 2011), no such effect was seen in liverspecific ABCA1 knockout mice on a LDLR-deficient background (Bi et al. 2013).

With respect to the final step of RCT, sterol excretion from the body, the differential contribution of the biliary pathway and the intestine is a subject of active study; it might be important to note that no unequivocally established concept has emerged thus far. The classical view on the RCT pathway puts biliary secretion of RCT-relevant cholesterol central (Annema and Tietge 2012). This view is supported by studies using either surgical disruption of biliary secretion or Abcb4 knockout mice that have a genetic defect in cholesterol secretion into the bile secondary to their inability to secrete phospholipids and form mixed micelles (Nijstad et al. 2011). In the surgical model RCT was virtually absent, while in the genetic model, RCT was strongly reduced (Nijstad et al. 2011). Studies in ezetimibe-treated mice that express NPC1L1 only in hepatocytes reached a similar conclusion, namely, that functional RCT in this model depended on efficient biliary sterol secretion (Xie et al. 2013). On the other hand, RCT studies in NPC1L1 livertransgenic mice on a wild-type background as well as a short-term experiment in bile-diverted mice resulted in opposite findings, namely, that RCT can proceed when the biliary secretion pathway is impaired (Temel et al. 2010). Further studies are clearly required to assess the role of the intestine and a possible contribution of TICE to macrophage RCT. However, currently such experiments are hampered by the lack of information how TICE is precisely mediated (Tietge and Groen 2013). Commonly accepted on the other hand is the role of intestinal sterol absorption in $\mathrm{RCT}$, and several pharmacological intervention studies were carried out to show that blocking sterol absorption increases RCT (for details please see Table 1 and text above).

In addition, there are also more complex systemic pathophysiological states that are clinically associated with an increased atherosclerosis incidence and in which also alterations in RCT have been observed in mouse models. One example is an inflammatory response. Consistently, RCT was severely impaired in LPS- (Annema et al. 2010; McGillicuddy et al. 2009) and zymosan-induced (Malik et al. 2011) models of acute inflammation. Another example is diabetes. In both, an insulindeficient model of type 1 diabetes (de Boer et al. 2012) as well as in $\mathrm{db} / \mathrm{db}$ mice (Low et al. 2012), which lack the leptin receptor and serve as model of type 2 diabetes, RCT was decreased. Interestingly, the first study indicated that the selective uptake step of HDL into the liver is defective in type 1 diabetic mice (de Boer et al. 2012), while the latter study provided evidence that advanced glycation end products are not likely to be pathophysiologically involved (Low et al. 2012). Furthermore, acute psychological stress has been shown to increase 


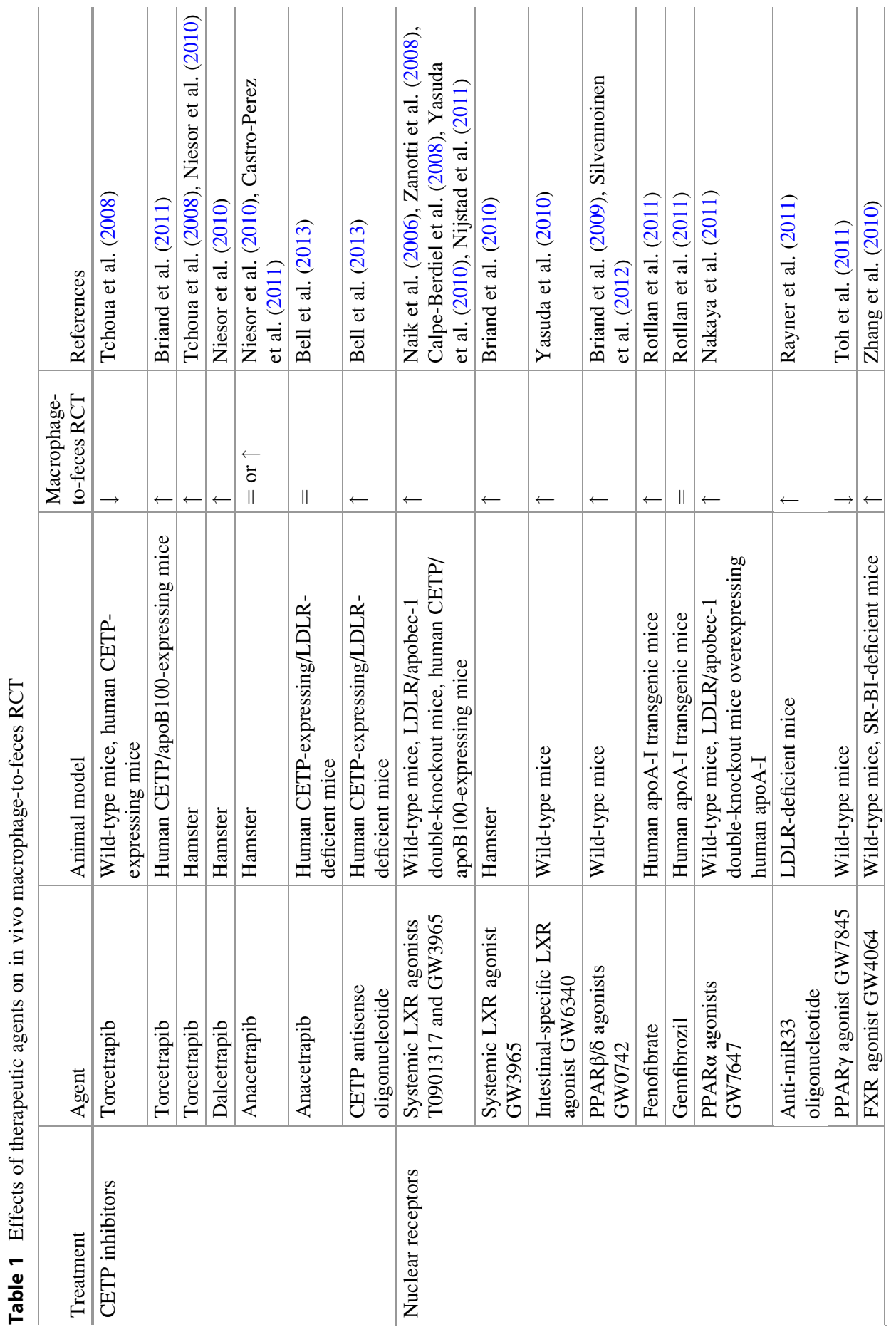




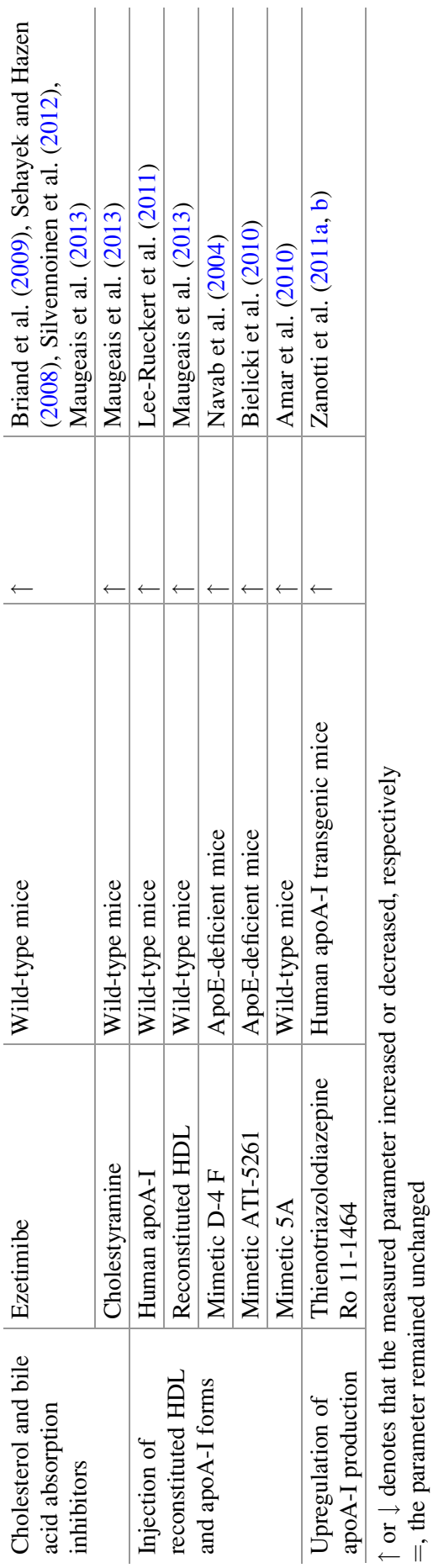


RCT in mice, mainly by a corticosterone-mediated downregulation of NPC1L1 and subsequent reduction in intestinal cholesterol absorption (Silvennoinen et al. 2012). These data underline the important role of intestinal NPC1L1 in RCT but are counterintuitive to the increased atherosclerosis risk attributed to psychological stress in clinical settings.

Finally, two recent additions of pathways that are linked to RCT have emerged which are not so obvious given the classical view on the RCT pathway but are worth mentioning. On the one hand, red blood cells were shown to contribute to RCT in a way that functional RCT was decreased in anemic mice (Hung et al. 2012). On the other hand, lymphatic drainage has been indicated to be involved in RCT (Martel et al. 2013; Lim et al. 2013). Hereby, blocking lymphatic transport or lymph vessel regrowth inhibited RCT, while enabling it increased RCT (Martel et al. 2013; Lim et al. 2013). Interestingly, for this route of RCT, transcytosis of HDL through lymphatic endothelium in a SR-BI-dependent fashion was critical (Lim et al. 2013), findings very similar to the role of SR-BI in HDL transcytosis through vascular endothelial cells (Rohrer et al. 2009).

\subsection{Pharmacology}

RCT-enhancing therapies are currently considered a promising strategy for the prevention and treatment of atherosclerotic CVD. An important number of RCT-targeted drugs have been used in preclinical animal models (mainly mice and hamsters) to test their effects on in vivo RCT from labeled cholesterol macrophages to feces. These RCT-targeted drugs can be classified among four different therapeutic approaches: CETP inhibition, nuclear receptors activation, cholesterol absorption inhibition, and directly augmenting or mimicking apoA-I. Most of these drugs are being used in clinical practice or tested in clinical trials in phases I, II, or III. This section discusses recent findings indicating that some of these therapies may be atheroprotective by promoting RCT in vivo (Table 1 shows a summary of available data).

\subsubsection{CETP Inhibitors}

CETP inhibition presents a preferential target for raising HDL-C and enhancing RCT. However, available data on the effect of the CETP inhibitors torcetrapib, dalcetrapib, and anacetrapib in macrophage-to-feces RCT have produced divergent results (Tchoua et al. 2008; Briand et al. 2011; Niesor et al. 2010; Castro-Perez et al. 2011). These controversial effects, together with the disappointing results of two large clinical trials using torcetrapib and dalcetrapib, have raised reasonable doubts regarding the clinical use of CETP inhibitors (Barter and Rye 2012). The positive effects of CETP antisense oligonucleotides on macrophage RCT (Bell et al. 2013) open up an alternative pathway to further evaluate whether the inhibition of CETP may improve cardiovascular risk. 


\subsubsection{Nuclear Receptor Activation}

Liver X receptor (LXR) is considered an attractive target for therapeutic strategies aimed at stimulating RCT since it promotes HDL biogenesis, macrophage cholesterol efflux, and biliary cholesterol excretion. A considerable number of studies tested the effect of systemic LXR agonists T0901317 and GW3965 on macrophageto-feces RCT and consistently found a higher flux through this pathway (Naik et al. 2006; Zanotti et al. 2008; Calpe-Berdiel et al. 2008; Yasuda et al. 2010; Nijstad et al. 2011; Briand et al. 2010). However, systemic LXR activators have detrimental consequences for the liver such as the induction of lipogenesis. As discussed in Sect. 3.1.2, multiple evidences indicate that excretion of macrophagederived cholesterol can be modulated in the last step of RCT pathway which occurs in the small intestine. Indeed, intestine-specific LXR activator GW6340 also enhances macrophage RCT but avoids the lipogenic toxicity associated with liver LXR activation in mice (Yasuda et al. 2010). Peroxisome proliferator-activated receptor (PPAR) $\alpha$ agonists such as GW7647 and fenofibrate promote macrophage RCT (Rotllan et al. 2011; Nakaya et al. 2011). This effect is strongly correlated with the positive effects on apoA-I levels; more importantly, macrophage PPAR $\alpha$ and LXR expression are required for the PPAR $\alpha$-mediated enhancement of macrophage RCT (Nakaya et al. 2011). ABCA1 and G1 are targeted for degradation by microRNA (miR)-33, an intronic microRNA located within the SREBF2 gene; anti-miR-33 therapy enhances macrophage RCT (Rayner et al. 2011). In contrast, the PPAR $\gamma$ agonist GW7845 reduces macrophage RCT. The authors hypothesize that GW7845 redirects macrophage-derived cholesterol to adipose tissue via SR-BI, thereby reducing its biliary excretion (Toh et al. 2011). Beyond PPARLXR activation, the farnesoid X receptor (FXR) agonist GW4064 also promotes macrophage-to-feces RCT; this effect is related to liver SR-BI upregulation and reduced intestinal cholesterol absorption (Zhang et al. 2010).

\subsubsection{Cholesterol Absorption Inhibitors}

Interventions that inhibit cholesterol absorption including ezetimibe administration and PPAR $\beta / \delta$ activation with GW0742 increase the excretion of macrophagederived cholesterol in feces by reducing intestinal NPC1L1 activity (Briand et al. 2009; Sehayek and Hazen 2008; Silvennoinen et al. 2012; Maugeais et al. 2013). The bile acid sequestrant cholestyramine also promotes macrophageto-feces RCT (Maugeais et al. 2013).

\subsubsection{Augmenting or Mimicking apoA-I}

The use of apoA-I and its different forms for the prevention and treatment of atherosclerosis is a long-term goal of many laboratories. The full-length apoA-I, reconstituted apoA-I-containing HDL, or apoA-I mimetics D4-F, ATI-5261, and $5 \mathrm{~A}$ have been demonstrated to be effective for enhancing macrophage-to-feces RCT in different mouse models (Maugeais et al. 2013; Lee-Rueckert and Kovanen 2011; Navab et al. 2004; Bielicki et al. 2010; Amar et al. 2010). The upregulation of liver apoA-I production, as occurred with the thienotriazolodiazepine Ro 11-1464, also enhances macrophage RCT (Zanotti et al. 2011a, b). 


\section{Serum Cholesterol Efflux Capacity (CEC)}

Several epidemiological studies define HDL as the most powerful plasmatic factor with atheroprotective activity in humans (Di Angelantonio et al. 2009); a $1 \mathrm{mg} / \mathrm{dl}$ increase of plasma HDL-C is associated with a 3-4\% reduction in cardiovascular mortality (Assmann et al. 2002). Some post hoc analyses from randomized controlled trials also suggest that raising HDL-C beneficially affects the risk of CVD (Toth et al. 2013). However, the clinical efficacy of raising plasma HDL-C levels to achieve cardiovascular risk reduction has been difficult to prove. Recently published outcome trials involving the addition of niacin or dalcetrapib to standard low-density lipoprotein cholesterol reduction therapy failed to demonstrate clinical benefit despite increases in HDL-C ((AIM-HIGH) trial 2011; Schwartz et al. 2012). Furthermore, genetic variants associated with increased HDL-C, thus conferring lifelong exposure to higher circulating levels, are not consistently associated with improved vascular outcomes (Voight et al. 2012). These findings have reinforced the idea that changes in HDL-C levels are an inadequate surrogate for therapeutic use. Therefore, an emerging concept is that of the quality of HDL, which are heterogeneous in terms of size, charge, and lipid content (Calabresi et al. 2010) and display functional differences, such as cell cholesterol efflux promotion. Animal studies have suggested that HDL-mediated cholesterol flux through the different RCT steps is a better predictor of the atherosclerotic impact of various genetic and pharmacological perturbations than static, mass-based quantification of circulating HDL-C (Rader et al. 2009). The efficacy of such HDL function in a single individual may be estimated by measuring the cholesterol efflux capacity (CEC) using widely standardized techniques that allow distinguishing between the various mechanisms involved (Adorni et al. 2007). Several lines of evidence suggest that CEC is sensitive to HDL composition rather than HDL-C plasma levels; for instance, it has been shown that the serum capacity to promote cholesterol efflux via ABCA1 strictly depends on the nascent (pre $\beta$ ) HDL plasma levels (de la Llera-Moya et al. 2010; Favari et al. 2004). Subjects with the apoA-I Milano $_{\text {an }}$ mutation or LCAT deficiency have high levels of circulating particles and efficient serum ability to induce macrophage cholesterol depletion despite very low HDL levels (Favari et al. 2007; Calabresi et al. 2009). Thus, there is increasing evidence that the measure of HDL functionality in different populations may be a better predictor of coronary artery disease than the measure of absolute HDL-C levels (Khera et al. 2011). As mentioned before, this concept is consistent with studies revealing that subjects affected by CVD not only have HDL deficiency but also major rearrangements of their composition (Campos et al. 1995; Sweetnam et al. 1994) and consequently function. The role of serum CEC as an index of cardiovascular protection has been suggested by a recent study demonstrating that in two distinct cohorts of subjects, the CEC variable has a stronger predictive power of the carotid intima-media thickness, an index of subclinical atherosclerosis, than plasma HDL-C levels (Khera et al. 2011). In addition, in a population of healthy subjects, ABCA1-mediated serum CEC was inversely correlated with pulse wave velocity, an index of arterial stiffness, independent of HDL-C serum levels (Favari 
et al. 2013). A work measuring the flow-mediated dilation, as a parameter of endothelial function, showed a positive correlation with the ABCA1-mediated efflux pathway confirming the concept that the functional measures of HDL might be a better marker for cardiovascular risk rather than HDL cholesterol levels (Vazquez et al. 2012). Recently, Li and colleagues provided data indicating that individuals in the top tertile of CEC had a moderately increased risk of a composite cardiovascular end point of incident myocardial infarction, stroke, or death during 3 years of follow-up ( $\mathrm{Li}$ et al. 2013). However, in the same study, CEC was inversely associated with coronary artery disease. The reasons for this apparently contradictory findings require further evaluation, but may be related to the characteristics of the population involved in the study (Khera and Rader 2013). Overall, the available data suggest that evaluation of CEC may better correlate with coronary artery diseases than HDL-C; however, further studies are required to demonstrate its role as predictor of cardiovascular event risk.

\section{Conclusions}

RCT provides a physiological strategy of protection from atherosclerosis. HDL plays a leading role by promoting the removal of excess cholesterol in the arterial wall through the induction of cellular cholesterol efflux from cells. Cholesterol efflux represents the first and perhaps most important step of RCT. The ability of HDL to promote cholesterol efflux depends on their quality and the pathologic processes that induce their modifications. Many of the available data on the physiology and pharmacology of RCT depend on studies in animal models that have demonstrated that the enhancement of RCT is inversely correlated with the development of atherosclerosis. In humans, the efficiency of the RCT can be evaluated with the surrogate parameter of serum cholesterol efflux capacity (CEC) that indicates the ability of HDL to promote efflux of cholesterol in the individual patient. Recent clinical data suggest that the evaluation of CEC is a strong predictor of atherosclerosis extent in humans and may represent in the future a useful biomarker of cardiovascular risk.

Open Access This chapter is distributed under the terms of the Creative Commons Attribution Noncommercial License, which permits any noncommercial use, distribution, and reproduction in any medium, provided the original author(s) and source are credited.

\section{References}

Adorni MP, Zimetti F et al (2007) The roles of different pathways in the release of cholesterol from macrophages. J Lipid Res 48(11):2453-2462

Adorni MP, Favari E et al (2011) Free cholesterol alters macrophage morphology and mobility by an ABCA1 dependent mechanism. Atherosclerosis 215(1):70-76

AIM-HIGH (2011) The role of niacin in raising high-density lipoprotein cholesterol to reduce cardiovascular events in patients with atherosclerotic cardiovascular disease and optimally treated low-density lipoprotein cholesterol: baseline characteristics of study participants. The 
Atherothrombosis Intervention in Metabolic syndrome with low HDL/high triglycerides: impact on Global Health outcomes (AIM-HIGH) trial. Am Heart J 161(3):538-543

Amar MJ, D'Souza W et al (2010) 5A apolipoprotein mimetic peptide promotes cholesterol efflux and reduces atherosclerosis in mice. J Pharmacol Exp Ther 334(2):634-641

Annema W, Tietge UJ (2011) Role of hepatic lipase and endothelial lipase in high-density lipoprotein-mediated reverse cholesterol transport. Curr Atheroscler Rep 13(3):257-265

Annema W, Tietge UJ (2012) Regulation of reverse cholesterol transport - a comprehensive appraisal of available animal studies. Nutr Metab (Lond) 9(1):25

Annema W, von Eckardstein A (2013) High-density lipoproteins. Multifunctional but vulnerable protections from atherosclerosis. Circ J 77(10):2432-2448

Annema W, Nijstad N et al (2010) Myeloperoxidase and serum amyloid A contribute to impaired in vivo reverse cholesterol transport during the acute phase response but not group IIA secretory phospholipase A(2). J Lipid Res 51(4):743-754

Annema W, Dikkers A et al (2012) ApoE promotes hepatic selective uptake but not RCT due to increased ABCA1-mediated cholesterol efflux to plasma. J Lipid Res 53(5):929-940

Assmann G, Cullen P et al (2002) Simple scoring scheme for calculating the risk of acute coronary events based on the 10-year follow-up of the prospective cardiovascular Munster (PROCAM) study. Circulation 105(3):310-315

Barter PJ, Rye KA (2012) Cholesteryl ester transfer protein inhibition as a strategy to reduce cardiovascular risk. J Lipid Res 53(9):1755-1766

Bell TA 3rd, Graham MJ et al (2013) Antisense oligonucleotide inhibition of cholesteryl ester transfer protein enhances RCT in hyperlipidemic, CETP transgenic, LDLr-/- mice. J Lipid Res 54(10):2647-2657

Bergt C, Pennathur S et al (2004) The myeloperoxidase product hypochlorous acid oxidizes HDL in the human artery wall and impairs ABCA1-dependent cholesterol transport. Proc Natl Acad Sci U S A 101(35):13032-13037

Bi X, Zhu X et al (2013) Liver ABCA1 deletion in LDLrKO mice does not impair macrophage reverse cholesterol transport or exacerbate atherogenesis. Arterioscler Thromb Vasc Biol 33 (10):2288-2296

Bielicki JK, Zhang H et al (2010) A new HDL mimetic peptide that stimulates cellular cholesterol efflux with high efficiency greatly reduces atherosclerosis in mice. J Lipid Res 51(6): 1496-1503

Boadu E, Francis GA (2006) The role of vesicular transport in ABCA1-dependent lipid efflux and its connection with NPC pathways. J Mol Med (Berl) 84(4):266-275

Briand F, Naik SU et al (2009) Both the peroxisome proliferator-activated receptor delta agonist, GW0742, and ezetimibe promote reverse cholesterol transport in mice by reducing intestinal reabsorption of HDL-derived cholesterol. Clin Transl Sci 2(2):127-133

Briand F, Treguier M et al (2010) Liver X receptor activation promotes macrophage-to-feces reverse cholesterol transport in a dyslipidemic hamster model. J Lipid Res 51(4):763-770

Briand F, Thieblemont Q et al (2011) CETP inhibitor torcetrapib promotes reverse cholesterol transport in obese insulin-resistant CETP-ApoB100 transgenic mice. Clin Transl Sci 4(6): 414-420

Brown RJ, Lagor WR et al (2010) Impact of combined deficiency of hepatic lipase and endothelial lipase on the metabolism of both high-density lipoproteins and apolipoprotein B-containing lipoproteins. Circ Res 107(3):357-364

Calabresi L, Favari E et al (2009) Functional LCAT is not required for macrophage cholesterol efflux to human serum. Atherosclerosis 204(1):141-146

Calabresi L, Gomaraschi M et al (2010) High-density lipoprotein quantity or quality for cardiovascular prevention? Curr Pharm Des 16(13):1494-1503

Calpe-Berdiel L, Rotllan N et al (2008) Liver X receptor-mediated activation of reverse cholesterol transport from macrophages to feces in vivo requires ABCG5/G8. J Lipid Res 49(9): 1904-1911 
Campos H, Roederer GO et al (1995) Predominance of large LDL and reduced HDL2 cholesterol in normolipidemic men with coronary artery disease. Arterioscler Thromb Vasc Biol 15(8): 1043-1048

Castro-Perez J, Briand F et al (2011) Anacetrapib promotes reverse cholesterol transport and bulk cholesterol excretion in Syrian golden hamsters. J Lipid Res 52(11):1965-1973

Cavigiolio G, Geier EG et al (2010) Exchange of apolipoprotein A-I between lipid-associated and lipid-free states: a potential target for oxidative generation of dysfunctional high density lipoproteins. J Biol Chem 285(24):18847-18857

Charles-Schoeman C, Lee YY et al (2012) Cholesterol efflux by high density lipoproteins is impaired in patients with active rheumatoid arthritis. Ann Rheum Dis 71(7):1157-1162

Chroni A, Liu T et al (2003) The central helices of ApoA-I can promote ATP-binding cassette transporter A1 (ABCA1)-mediated lipid efflux. Amino acid residues 220-231 of the wild-type ApoA-I are required for lipid efflux in vitro and high density lipoprotein formation in vivo. J Biol Chem 278(9):6719-6730

Chroni A, Liu T et al (2004) Cross-linking and lipid efflux properties of apoA-I mutants suggest direct association between apoA-I helices and ABCA1. Biochemistry 43(7):2126-2139

Chroni A, Koukos G et al (2007) The carboxy-terminal region of apoA-I is required for the ABCA1-dependent formation of alpha-HDL but not prebeta-HDL particles in vivo. Biochemistry 46(19):5697-5708

Daniil G, Zannis VI et al (2013) Effect of apoA-I mutations in the capacity of reconstituted HDL to promote ABCG1-mediated cholesterol efflux. PLoS ONE 8(6):e67993

Dawson PA (2011) Role of the intestinal bile acid transporters in bile acid and drug disposition. Handb Exp Pharmacol 201:169-203

de Boer JF, Annema W et al (2012) Type I diabetes mellitus decreases in vivo macrophage-tofeces reverse cholesterol transport despite increased biliary sterol secretion in mice. J Lipid Res 53(3):348-357

de la Llera-Moya M, Drazul-Schrader D et al (2010) The ability to promote efflux via ABCA1 determines the capacity of serum specimens with similar high-density lipoprotein cholesterol to remove cholesterol from macrophages. Arterioscler Thromb Vasc Biol 30(4):796-801

Di Angelantonio E, Sarwar N et al (2009) Major lipids, apolipoproteins, and risk of vascular disease. JAMA 302(18):1993-2000

Dikkers A, Tietge UJ (2010) Biliary cholesterol secretion: more than a simple ABC. World J Gastroenterol 16(47):5936-5945

Dikkers A, Freak de Boer J et al (2013) Scavenger receptor BI and ABCG5/G8 differentially impact biliary sterol secretion and reverse cholesterol transport in mice. Hepatology 58(1): 293-303

Dunbar RL, Cuchel M, Millar JS, Baer A, Poria R, Freifelder RH, Pryma DA, Billheimer JJ, Rader DJ (2013) Abstract 439: niacin does not accelerate reverse cholesterol transport in man. Arterioscler Thromb Vasc Biol 33:A439

Eberini I, Calabresi L et al (2002) Macrophage metalloproteinases degrade high-density-lipoprotein-associated apolipoprotein A-I at both the N- and C-termini. Biochem J 362(Pt 3):627-634

El Bouhassani M, Gilibert S et al (2011) Cholesteryl ester transfer protein expression partially attenuates the adverse effects of SR-BI receptor deficiency on cholesterol metabolism and atherosclerosis. J Biol Chem 286(19):17227-17238

Escola-Gil JC, Chen X et al (2013) Hepatic lipase- and endothelial lipase-deficiency in mice promotes macrophage-to-feces RCT and HDL antioxidant properties. Biochim Biophys Acta 1831(4):691-697

Fabre AC, Malaval C et al (2010) P2Y13 receptor is critical for reverse cholesterol transport. Hepatology 52(4):1477-1483

Favari E, Bernini F et al (2002) The C-terminal domain of apolipoprotein A-I is involved in ABCA1-driven phospholipid and cholesterol efflux. Biochem Biophys Res Commun 299(5): 801-805 
Favari E, Lee M et al (2004) Depletion of pre-beta-high density lipoprotein by human chymase impairs ATP-binding cassette transporter A1- but not scavenger receptor class B type I-mediated lipid efflux to high density lipoprotein. J Biol Chem 279(11):9930-9936

Favari E, Gomaraschi M et al (2007) A unique protease-sensitive high density lipoprotein particle containing the apolipoprotein A-I(Milano) dimer effectively promotes ATP-binding cassette A1-mediated cell cholesterol efflux. J Biol Chem 282(8):5125-5132

Favari E, Calabresi L et al (2009) Small discoidal pre-beta1 HDL particles are efficient acceptors of cell cholesterol via ABCA1 and ABCG1. Biochemistry 48(46):11067-11074

Favari E, Ronda N et al (2013) ABCA1-dependent serum cholesterol efflux capacity inversely correlates with pulse wave velocity in healthy subjects. J Lipid Res 54(1):238-243

Freeman SR, Jin X et al (2013) ABCG1-mediated generation of extracellular cholesterol microdomains. J Lipid Res 55(1):115-127

Fu Y, Mukhamedova N et al (2013) ABCA12 regulates ABCA1-dependent cholesterol efflux from macrophages and the development of atherosclerosis. Cell Metab 18(2):225-238

Gargalovic P, Dory L (2003) Caveolins and macrophage lipid metabolism. J Lipid Res 44(1): $11-21$

Gelissen IC, Harris M et al (2006) ABCA1 and ABCG1 synergize to mediate cholesterol export to apoA-I. Arterioscler Thromb Vasc Biol 26(3):534-540

Holzer M, Wolf P et al (2013) Anti-psoriatic therapy recovers high-density lipoprotein composition and function. J Invest Dermatol 134(3):635-642

Huang ZH, Lin CY et al (2001) Sterol efflux mediated by endogenous macrophage ApoE expression is independent of ABCA1. Arterioscler Thromb Vasc Biol 21(12):2019-2025

Huang ZH, Gu D et al (2003) Expression of scavenger receptor BI facilitates sterol movement between the plasma membrane and the endoplasmic reticulum in macrophages. Biochemistry 42(13):3949-3955

Hung KT, Berisha SZ et al (2012) Red blood cells play a role in reverse cholesterol transport. Arterioscler Thromb Vasc Biol 32(6):1460-1465

Ishimoto Y, Yamada K et al (2003) Group V and X secretory phospholipase A(2)s-induced modification of high-density lipoprotein linked to the reduction of its antiatherogenic functions. Biochim Biophys Acta 1642(3):129-138

Khera AV, Rader DJ (2013) Cholesterol efflux capacity: full steam ahead or a bump in the road? Arterioscler Thromb Vasc Biol 33(7):1449-1451

Khera AV, Cuchel M et al (2011) Cholesterol efflux capacity, high-density lipoprotein function, and atherosclerosis. N Engl J Med 364(2):127-135

Kockx M, Jessup W et al (2008) Regulation of endogenous apolipoprotein E secretion by macrophages. Arterioscler Thromb Vasc Biol 28(6):1060-1067

Kunitake ST, Chen GC et al (1990) Pre-beta high density lipoprotein. Unique disposition of apolipoprotein A-I increases susceptibility to proteolysis. Arteriosclerosis 10(1):25-30

Landry YD, Denis M et al (2006) ATP-binding cassette transporter A1 expression disrupts raft membrane microdomains through its ATPase-related functions. J Biol Chem 281(47): 36091-36101

Larrede S, Quinn CM et al (2009) Stimulation of cholesterol efflux by LXR agonists in cholesterol-loaded human macrophages is ABCA1-dependent but ABCG1-independent. Arterioscler Thromb Vasc Biol 29(11):1930-1936

Lee M, Kovanen PT et al (2003) Apolipoprotein composition and particle size affect HDL degradation by chymase: effect on cellular cholesterol efflux. J Lipid Res 44(3):539-546

Lee-Rueckert M, Kovanen PT (2011) Extracellular modifications of HDL in vivo and the emerging concept of proteolytic inactivation of prebeta-HDL. Curr Opin Lipidol 22(5): 394-402

Lee-Rueckert M, Silvennoinen R et al (2011) Mast cell activation in vivo impairs the macrophage reverse cholesterol transport pathway in the mouse. Arterioscler Thromb Vasc Biol 31(3): $520-527$ 
Li XM, Tang WH et al (2013) Paradoxical association of enhanced cholesterol efflux with increased incident cardiovascular risks. Arterioscler Thromb Vasc Biol 33(7):1696-1705

Lichtenstein L, Serhan N et al (2013) Lack of P2Y13 in mice fed a high cholesterol diet results in decreased hepatic cholesterol content, biliary lipid secretion and reverse cholesterol transport. Nutr Metab (Lond) 10(1):67

Lim HY, Thiam CH et al (2013) Lymphatic vessels are essential for the removal of cholesterol from peripheral tissues by SR-BI-mediated transport of HDL. Cell Metab 17(5):671-684

Lindstedt L, Saarinen J et al (1999) Matrix metalloproteinases-3, -7, and -12, but not -9 , reduce high density lipoprotein-induced cholesterol efflux from human macrophage foam cells by truncation of the carboxyl terminus of apolipoprotein A-I. Parallel losses of pre-beta particles and the high affinity component of efflux. J Biol Chem 274(32):22627-22634

Low H, Hoang A et al (2012) Advanced glycation end-products (AGEs) and functionality of reverse cholesterol transport in patients with type 2 diabetes and in mouse models. Diabetologia 55(9):2513-2521

Malik P, Smith JP (2009) A novel in vivo assay for reverse cholesterol transport. Arterioscler Thromb Vasc Biol 29:E46

Malik P, Berisha SZ et al (2011) Zymosan-mediated inflammation impairs in vivo reverse cholesterol transport. J Lipid Res 52(5):951-957

Martel C, Li W et al (2013) Lymphatic vasculature mediates macrophage reverse cholesterol transport in mice. J Clin Invest 123(4):1571-1579

Maugeais C, Annema W et al (2013) rHDL administration increases reverse cholesterol transport in mice, but is not additive on top of ezetimibe or cholestyramine treatment. Atherosclerosis 229(1):94-101

Maxfield FR, Tabas I (2005) Role of cholesterol and lipid organization in disease. Nature 438 (7068):612-621

McGillicuddy FC, de la Llera Moya M et al (2009) Inflammation impairs reverse cholesterol transport in vivo. Circulation 119(8):1135-1145

Naik SU, Wang X et al (2006) Pharmacological activation of liver X receptors promotes reverse cholesterol transport in vivo. Circulation 113(1):90-97

Nakaya K, Tohyama J et al (2011) Peroxisome proliferator-activated receptor-alpha activation promotes macrophage reverse cholesterol transport through a liver $\mathrm{X}$ receptor-dependent pathway. Arterioscler Thromb Vasc Biol 31(6):1276-1282

Navab M, Anantharamaiah GM et al (2004) Oral D-4 F causes formation of pre-beta high-density lipoprotein and improves high-density lipoprotein-mediated cholesterol efflux and reverse cholesterol transport from macrophages in apolipoprotein E-null mice. Circulation 109(25): 3215-3220

Niesor EJ, Magg C et al (2010) Modulating cholesteryl ester transfer protein activity maintains efficient pre-beta-HDL formation and increases reverse cholesterol transport. J Lipid Res 51(12):3443-3454

Nijstad N, Gautier T et al (2011) Biliary sterol secretion is required for functional in vivo reverse cholesterol transport in mice. Gastroenterology 140(3):1043-1051

Olivier M, Tanck MW et al (2012) Human ATP-binding cassette G1 controls macrophage lipoprotein lipase bioavailability and promotes foam cell formation. Arterioscler Thromb Vasc Biol 32(9):2223-2231

Onat A, Direskeneli H (2012) Excess cardiovascular risk in inflammatory rheumatic diseases: pathophysiology and targeted therapy. Curr Pharm Des 18(11):1465-1477

Oram JF, Lawn RM (2001) ABCA1. The gatekeeper for eliminating excess tissue cholesterol. J Lipid Res 42(8):1173-1179

Oram JF, Vaughan AM (2006) ATP-binding cassette cholesterol transporters and cardiovascular disease. Circ Res 99(10):1031-1043

Out R, Jessup W et al (2008) Coexistence of foam cells and hypocholesterolemia in mice lacking the ABC transporters A1 and G1. Circ Res 102(1):113-120 
Pennathur S, Bergt C et al (2004) Human atherosclerotic intima and blood of patients with established coronary artery disease contain high density lipoprotein damaged by reactive nitrogen species. J Biol Chem 279(41):42977-42983

Pirillo A, Uboldi P et al (2006) 15-Lipoxygenase-mediated modification of high-density lipoproteins impairs SR-BI- and ABCA1-dependent cholesterol efflux from macrophages. Biochim Biophys Acta 1761(3):292-300

Rader DJ, Alexander ET et al (2009) The role of reverse cholesterol transport in animals and humans and relationship to atherosclerosis. J Lipid Res 50(Suppl):S189-S194

Ramirez CM, Davalos A et al (2011) MicroRNA-758 regulates cholesterol efflux through posttranscriptional repression of ATP-binding cassette transporter A1. Arterioscler Thromb Vasc Biol 31(11):2707-2714

Rayner KJ, Suarez Y et al (2010) MiR-33 contributes to the regulation of cholesterol homeostasis. Science 328(5985):1570-1573

Rayner KJ, Sheedy FJ et al (2011) Antagonism of miR-33 in mice promotes reverse cholesterol transport and regression of atherosclerosis. J Clin Invest 121(7):2921-2931

Rohrer L, Ohnsorg PM et al (2009) High-density lipoprotein transport through aortic endothelial cells involves scavenger receptor BI and ATP-binding cassette transporter G1. Circ Res 104 (10): $1142-1150$

Ronda N, Favari E et al (2013) Impaired serum cholesterol efflux capacity in rheumatoid arthritis and systemic lupus erythematosus. Ann Rheum Dis 73(3):609-615

Rosenson RS, Hurt-Camejo E (2012) Phospholipase A2 enzymes and the risk of atherosclerosis. Eur Heart J 33(23):2899-2909

Rosenson RS, Brewer HB Jr et al (2012) Cholesterol efflux and atheroprotection: advancing the concept of reverse cholesterol transport. Circulation 125(15):1905-1919

Rotllan N, Calpe-Berdiel L et al (2008) CETP activity variation in mice does not affect two major HDL antiatherogenic properties: macrophage-specific reverse cholesterol transport and LDL antioxidant protection. Atherosclerosis 196(2):505-513

Rotllan N, Llaverias G et al (2011) Differential effects of gemfibrozil and fenofibrate on reverse cholesterol transport from macrophages to feces in vivo. Biochim Biophys Acta 1811 (2): $104-110$

Samyn H, Moerland M et al (2009) Elevation of systemic PLTP, but not macrophage-PLTP, impairs macrophage reverse cholesterol transport in transgenic mice. Atherosclerosis 204(2): 429-434

Schmitz G, Langmann T et al (2001) Role of ABCG1 and other ABCG family members in lipid metabolism. J Lipid Res 42(10):1513-1520

Schwartz GG, Olsson AG et al (2012) Effects of dalcetrapib in patients with a recent acute coronary syndrome. N Engl J Med 367(22):2089-2099

Sehayek E, Hazen SL (2008) Cholesterol absorption from the intestine is a major determinant of reverse cholesterol transport from peripheral tissue macrophages. Arterioscler Thromb Vasc Biol 28(7):1296-1297

Shao B, Bergt C et al (2005) Tyrosine 192 in apolipoprotein A-I is the major site of nitration and chlorination by myeloperoxidase, but only chlorination markedly impairs ABCA1-dependent cholesterol transport. J Biol Chem 280(7):5983-5993

Shao B, Oda MN et al (2006) Myeloperoxidase: an inflammatory enzyme for generating dysfunctional high density lipoprotein. Curr Opin Cardiol 21(4):322-328

Silvennoinen R, Escola-Gil JC et al (2012) Acute psychological stress accelerates reverse cholesterol transport via corticosterone-dependent inhibition of intestinal cholesterol absorption. Circ Res 111(11):1459-1469

Sun D, Zhang J et al (2012) MiR-26 controls LXR-dependent cholesterol efflux by targeting ABCA1 and ARL7. FEBS Lett 586(10):1472-1479

Sweetnam PM, Bolton CH et al (1994) Associations of the HDL2 and HDL3 cholesterol subfractions with the development of ischemic heart disease in British men. The Caerphilly and Speedwell collaborative heart disease studies. Circulation 90(2):769-774 
Tanigawa H, Billheimer JT et al (2007) Expression of cholesteryl ester transfer protein in mice promotes macrophage reverse cholesterol transport. Circulation 116(11):1267-1273

Tanigawa H, Billheimer JT et al (2009) Lecithin: cholesterol acyltransferase expression has minimal effects on macrophage reverse cholesterol transport in vivo. Circulation 120(2): 160-169

Tarling EJ, Edwards PA (2012) Dancing with the sterols: critical roles for ABCG1, ABCA1, miRNAs, and nuclear and cell surface receptors in controlling cellular sterol homeostasis. Biochim Biophys Acta 1821(3):386-395

Tchoua U, D'Souza W et al (2008) The effect of cholesteryl ester transfer protein overexpression and inhibition on reverse cholesterol transport. Cardiovasc Res 77(4):732-739

Temel RE, Sawyer JK et al (2010) Biliary sterol secretion is not required for macrophage reverse cholesterol transport. Cell Metab 12(1):96-102

Tietge UJ, Groen AK (2013) Role the TICE?: advancing the concept of transintestinal cholesterol excretion. Arterioscler Thromb Vasc Biol 33(7):1452-1453

Timmins JM, Lee JY et al (2005) Targeted inactivation of hepatic Abca1 causes profound hypoalphalipoproteinemia and kidney hypercatabolism of apoA-I. J Clin Invest 115(5): $1333-1342$

Toh SA, Millar JS et al (2011) PPARgamma activation redirects macrophage cholesterol from fecal excretion to adipose tissue uptake in mice via SR-BI. Biochem Pharmacol 81(7):934-941

Toth PP, Barter PJ et al (2013) High-density lipoproteins: a consensus statement from the national lipid association. J Clin Lipidol 7(5):484-525

Usami Y, Matsuda K et al (2011) Detection of chymase-digested C-terminally truncated apolipoprotein A-I in normal human serum. J Immunol Methods 369(1-2):51-58

Usami Y, Kobayashi Y, Kameda T, Miyazaki A, Matsuda K, Sugano M, Kawasaki K, Kurihara Y, Kasama T, Tozuka M (2013) Identification of sites in apolipoprotein A-I susceptible to chymase and carboxypeptidase A digestion. Biosci Rep 33:49-56

Vantourout P, Radojkovic C et al (2010) Ecto-F(1)-ATPase: a moonlighting protein complex and an unexpected apoA-I receptor. World J Gastroenterol 16(47):5925-5935

Vaughan AM, Oram JF (2005) ABCG1 redistributes cell cholesterol to domains removable by high density lipoprotein but not by lipid-depleted apolipoproteins. J Biol Chem 280(34): 30150-30157

Vazquez E, Sethi AA et al (2012) High-density lipoprotein cholesterol efflux, nitration of apolipoprotein A-I, and endothelial function in obese women. Am J Cardiol 109(4):527-532

Vickers KC, Remaley AT (2014) HDL and cholesterol: life after the divorce? J Lipid Res 55(1): $4-12$

Voight BF, Peloso GM et al (2012) Plasma HDL cholesterol and risk of myocardial infarction: a mendelian randomisation study. Lancet 380(9841):572-580

Wang MD, Franklin V et al (2007a) In vivo reverse cholesterol transport from macrophages lacking ABCA1 expression is impaired. Arterioscler Thromb Vasc Biol 27(8):1837-1842

Wang X, Collins HL et al (2007b) Macrophage ABCA1 and ABCG1, but not SR-BI, promote macrophage reverse cholesterol transport in vivo. J Clin Invest 117(8):2216-2224

Watanabe J, Charles-Schoeman C et al (2012) Proteomic profiling following immunoaffinity capture of high-density lipoprotein: association of acute-phase proteins and complement factors with proinflammatory high-density lipoprotein in rheumatoid arthritis. Arthritis Rheum 64(6):1828-1837

Weibel GL, Hayes S et al (2011) Novel in vivo method for measuring cholesterol mass flux in peripheral macrophages. Arterioscler Thromb Vasc Biol 31(12):2865-2871

Wynn TA, Chawla A et al (2013) Macrophage biology in development, homeostasis and disease. Nature 496(7446):445-455

Xie P, Jia L et al (2013) Ezetimibe inhibits hepatic Niemann-Pick C1-Like 1 to facilitate macrophage reverse cholesterol transport in mice. Arterioscler Thromb Vasc Biol 33(5): 920-925 
Yamamoto S, Tanigawa $\mathrm{H}$ et al (2011) Pharmacologic suppression of hepatic ATP-binding cassette transporter 1 activity in mice reduces high-density lipoprotein cholesterol levels but promotes reverse cholesterol transport. Circulation 124(12):1382-1390

Yasuda T, Grillot D et al (2010) Tissue-specific liver X receptor activation promotes macrophage reverse cholesterol transport in vivo. Arterioscler Thromb Vasc Biol 30(4):781-786

Yvan-Charvet L, Kling J et al (2010) Cholesterol efflux potential and antiinflammatory properties of high-density lipoprotein after treatment with niacin or anacetrapib. Arterioscler Thromb Vasc Biol 30(7):1430-1438

Zanotti I, Poti F et al (2008) The LXR agonist T0901317 promotes the reverse cholesterol transport from macrophages by increasing plasma efflux potential. J Lipid Res 49(5):954-960

Zanotti I, Maugeais C et al (2011a) The thienotriazolodiazepine Ro 11-1464 increases plasma apoA-I and promotes reverse cholesterol transport in human apoA-I transgenic mice. $\mathrm{Br} \mathbf{J}$ Pharmacol 164(6):1642-1651

Zanotti I, Pedrelli M et al (2011b) Macrophage, but not systemic, apolipoprotein E is necessary for macrophage reverse cholesterol transport in vivo. Arterioscler Thromb Vasc Biol 31(1):74-80

Zanotti I, Favari E et al (2012) Cellular cholesterol efflux pathways: impact on intracellular lipid trafficking and methodological considerations. Curr Pharm Biotechnol 13(2):292-302

Zhang Y, Zanotti I et al (2003) Overexpression of apolipoprotein A-I promotes reverse transport of cholesterol from macrophages to feces in vivo. Circulation 108(6):661-663

Zhang Y, Da Silva JR et al (2005) Hepatic expression of scavenger receptor class B type I (SR-BI) is a positive regulator of macrophage reverse cholesterol transport in vivo. J Clin Invest 115 (10):2870-2874

Zhang Y, Yin L et al (2010) Identification of novel pathways that control farnesoid X receptormediated hypocholesterolemia. J Biol Chem 285(5):3035-3043

Zhao Y, Pennings $M$ et al (2011) Hypocholesterolemia, foam cell accumulation, but no atherosclerosis in mice lacking ABC-transporter A1 and scavenger receptor BI. Atherosclerosis 218 (2):314-322

Zheng L, Nukuna B et al (2004) Apolipoprotein A-I is a selective target for myeloperoxidasecatalyzed oxidation and functional impairment in subjects with cardiovascular disease. J Clin Invest 114(4):529-541

Zheng L, Settle M et al (2005) Localization of nitration and chlorination sites on apolipoprotein A-I catalyzed by myeloperoxidase in human atheroma and associated oxidative impairment in ABCA1-dependent cholesterol efflux from macrophages. J Biol Chem 280(1):38-47 